\title{
Qubit based Single-Photon Sensors for Dark Matter Searches
}

\section{Project Synopsis: Quantum Bits for Dark Matter Detection}

This experimental program will adapt low-noise qubit readout techniques for use in particle physics experiments. As an end goal, this research will deliver a technical demonstration of low-noise quantum sensors, as well as the results of a proof-ofprinciple axion search in a narrow but previously unexamined mass range. This program would form the basis for a technical design report outlining a next-generation axion dark matter search.
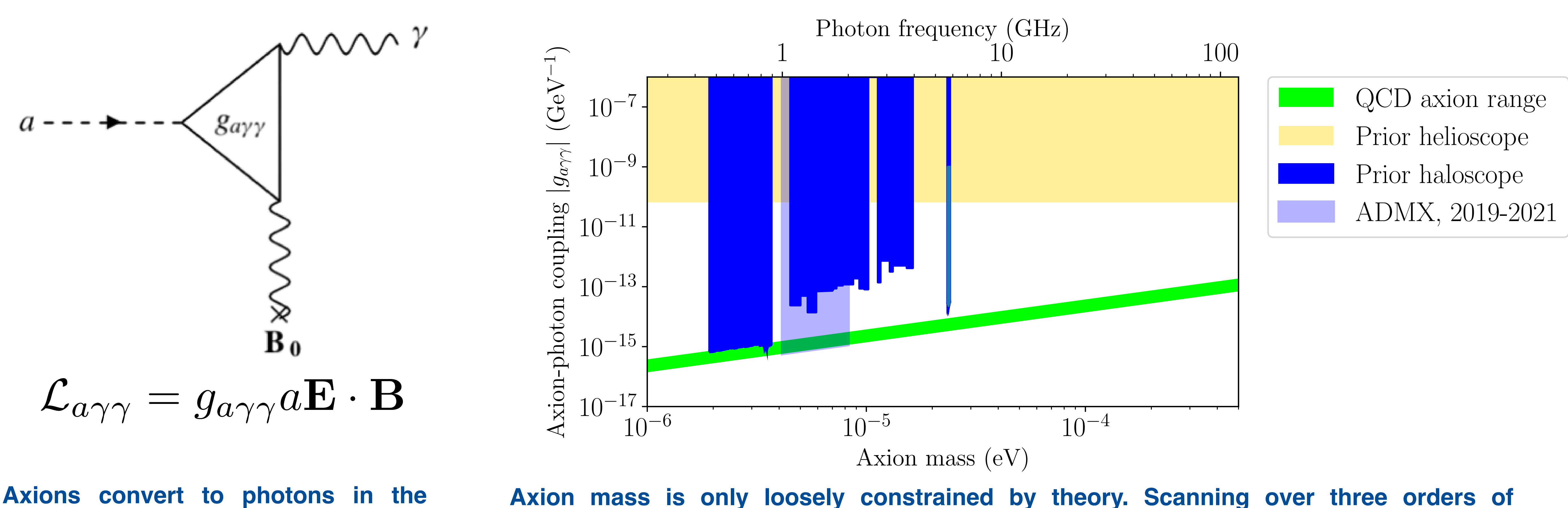

Axions convert to photons in the
presence of a magnetic field, with

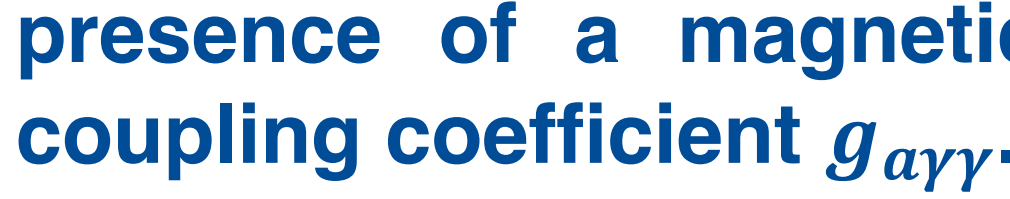

Axion mass is only loosely constrained by theory. Scanning over three orders of
magnitude in mass/frequency may be required. New technologies are required to perform magnitude in mass/frequ
this search efficiently.

Quantum non-demolition (QND) techniques allow repeated measurement of Fock states, to arbitrary precision.
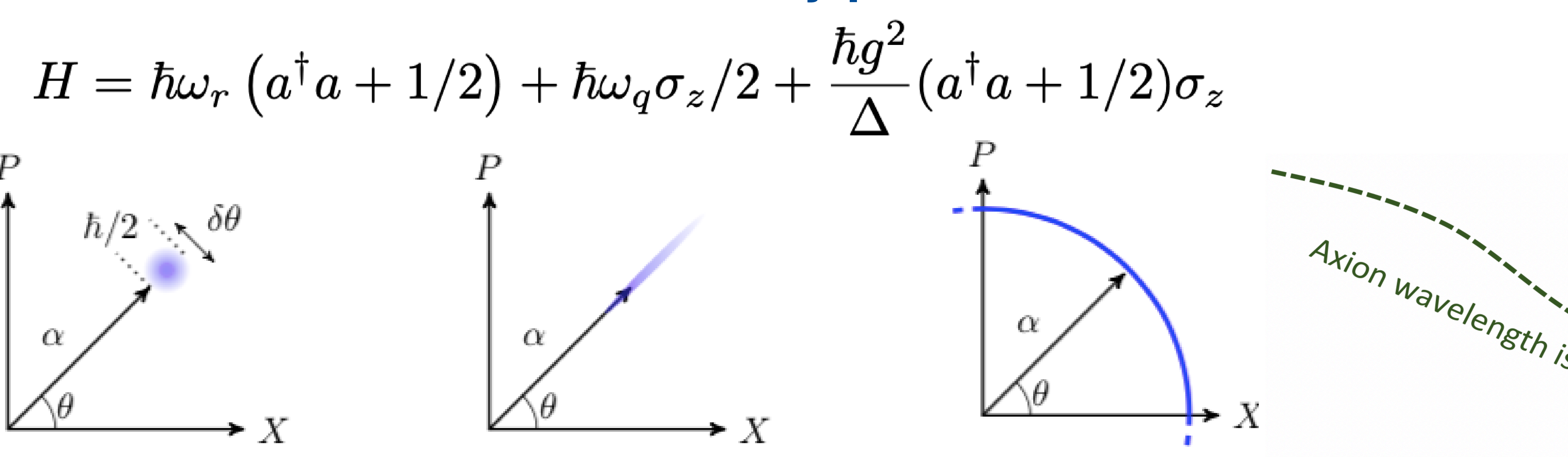

Even amplifiers operating at the quantum limit can be too noisy for efficient axion detection. Phase-preserving linear amplifiers simultaneously measure the occupation number and phase of a system, and these parameters have a nonzero commutator; they cannot be measured simultaneously to arbitrary precision. In a QND experiment, the phase of a photon state is randomized at every measurement so that amplitude (i.e. photon number) can be measured repeatedly and with high precision.

Quantum sensors enable low-noise photon sensitivity "beyond the standard quantum limit".

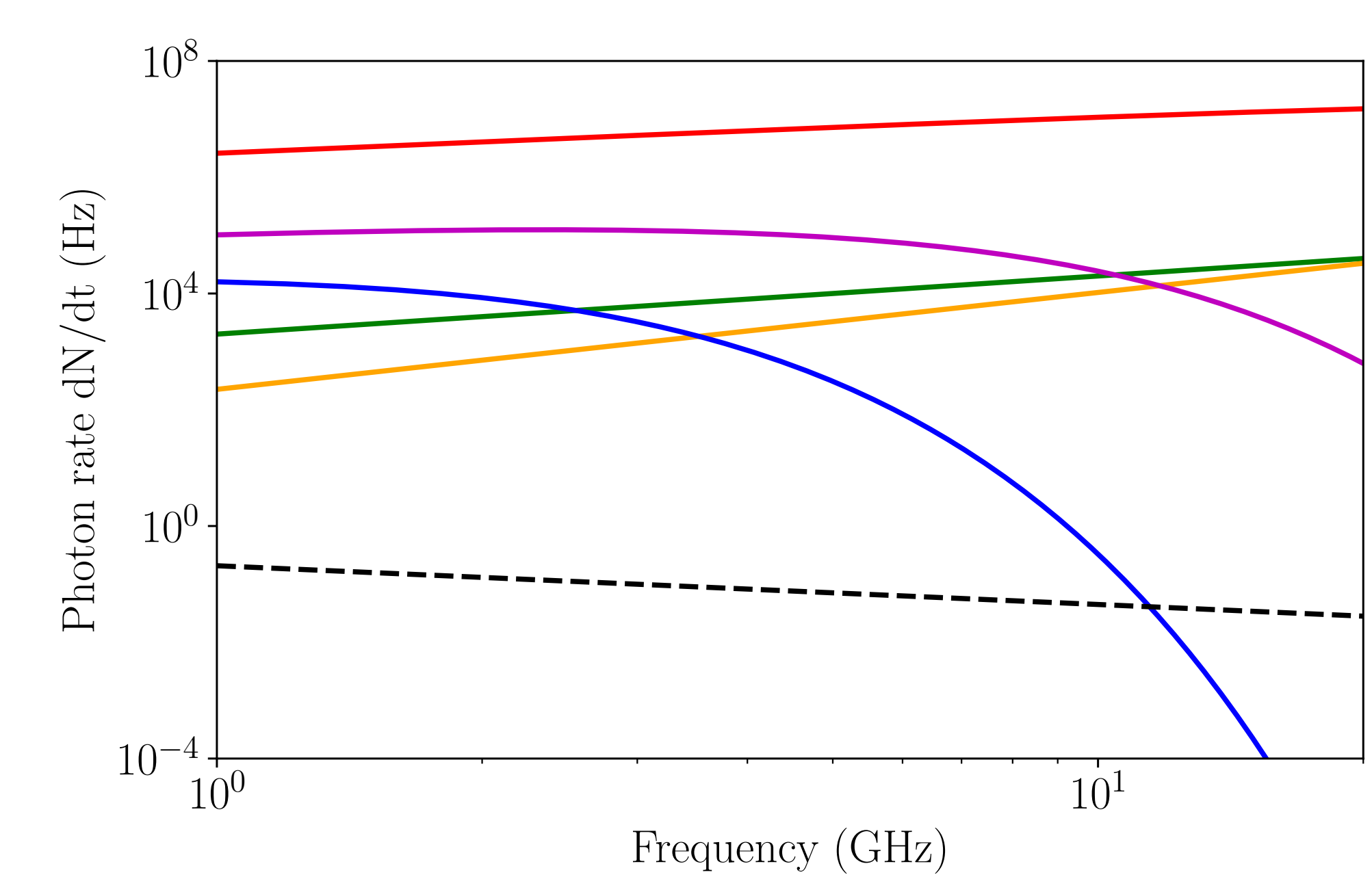

\section{- $\begin{gathered}\text { SQL } \\ \text { qubit error background, } p_{\operatorname{mer}}=0.01\end{gathered}$ — QND noise, $30 \mathrm{mK}$ - QND noise, $2 \mathrm{~K}$}

Expected signal rate (photons per second) for the DFSz family of axion models (black dashed line), compared with noise/error rates for phase-preserving linear quantum non-demolition (QND, blue/purple/red) measurements at various temperatures. The QND curves reflect thermal photon populations, since QND eliminates shot noise. The industry-typical qubit talse-positive error rate of $1 \%$ is

This measurement technique represents a novel application of quantum information technology to the field of particle physics. It has the potential to enhance axion search speeds by four orders of magnitude while enabling sensitivity to weak axion-photon coupling models.

\section{Current Work}

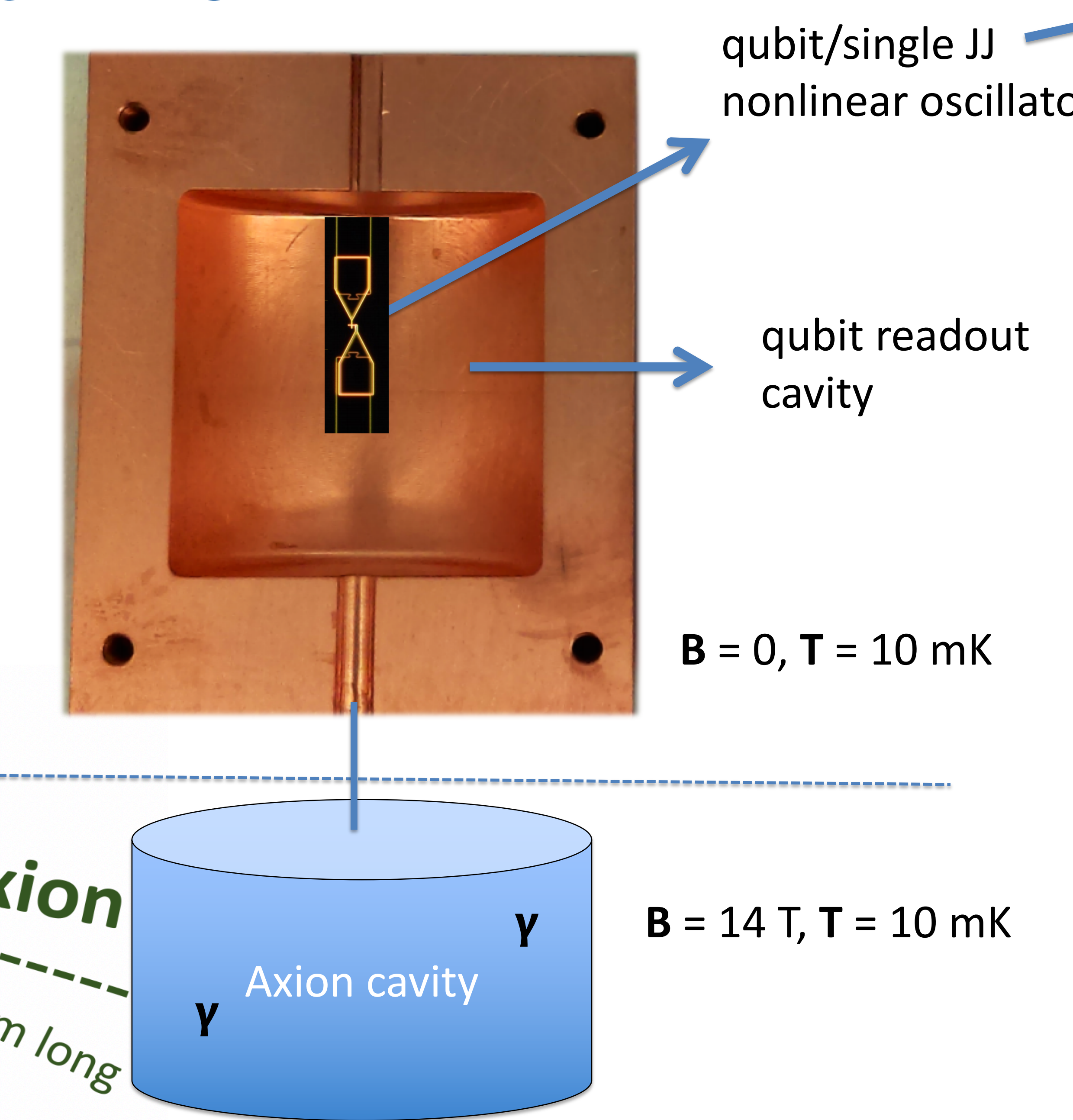

\section{Background Suppression}

false-positive rates for transmon qubits are $\sim 1 \%$. Several qubits, all measuring the same photon state can reduce this rate. For $\mathrm{N}$ qubits: $\boldsymbol{p}_{\text {err }} \rightarrow(\mathbf{0 . 0 1})^{N}$

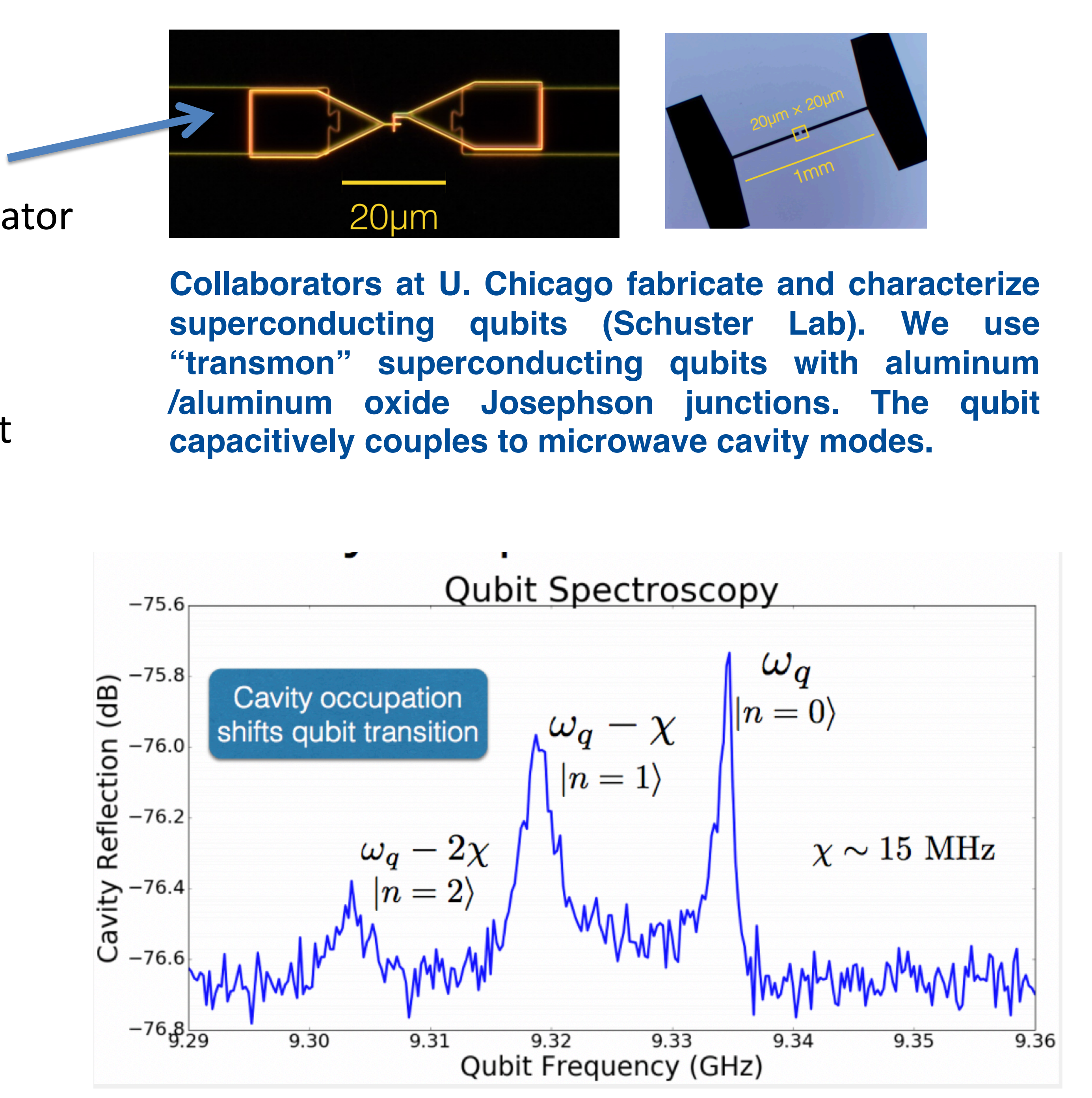

An AC Stark effect causes the cavity frequency A shitt by $(15 \mathrm{MHz})$ when a signal photon
interacts with the qubit. 